Supporting Information for

\title{
Fluffy Cotton-Like GO/Zn-Co-Ni Layered Double Hydroxides Form from a Sacrificed Template GO/ZIF-8 for High Performance Asymmetric Supercapacitors
}

\author{
Linli Zhu, Chen $\mathrm{Hao}^{*}$, Xiaohong Wang* and Yaning Guo \\ School of Chemistry and Chemical Engineering, Jiangsu University, Zhenjiang, \\ Jiangsu 212013, China
}

Number of pages: 13

Number of figures: 7

*Corresponding author. Tel.: +86 511 88791800; fax: +86 51188791800 .

E-mail addresses: chhao@ujs.edu.cn (C. Hao); xhwang@ujs.edu.cn (X.H. Wang). 


\section{Content}

Experimental Section

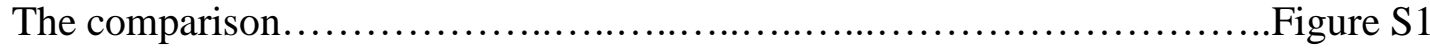

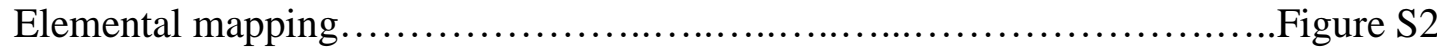

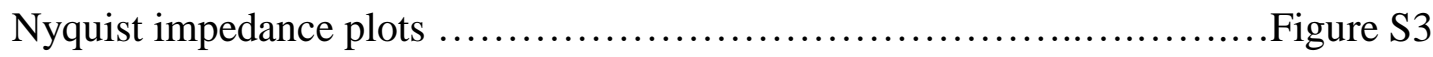

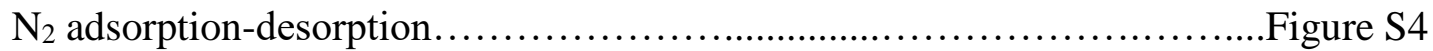

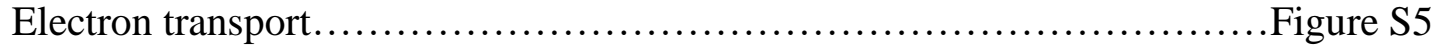

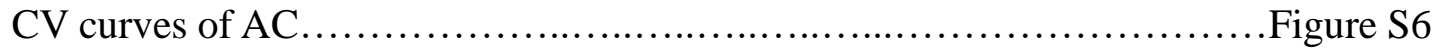

Nyquist impedance plots .......................................... Figure S7 


\section{Experimental section}

\section{Material and reagents}

All chemicals were of analytical grade and used without purification.

\section{Synthesis of GO/ZIF-8 composite}

$15 \mathrm{mg}$ GO synthesized by using a modified Hummers method was dissolved in 50 $\mathrm{mL}$ methanol followed by ultrasound for $1 \mathrm{~h}$ to form homogeneous solution. Then, $582 \mathrm{mg}$ zinc nitrate hexahydrate was weighed and added into the above solution. After ultrasound for another $1 \mathrm{~h}, 50 \mathrm{~mL}$ of methanol solution containing $656 \mathrm{mg}$ of 2-methyl imidazole was slowly and gradually dript into the above mixture at room temperature under magnetic stirring. After finishing the addition, another 30 minutes were spended on magnetic stirring and then the well mixed solution was aged for $24 \mathrm{~h}$ at room temperature. The last step was that the precursor sample was collected by centrifugation, washed alternately with water and ethanol for several times, and dried at $60{ }^{\circ} \mathrm{C}$ for $12 \mathrm{~h}$.

For comparison, the same qualities of 2-methylimidazole and zinc nitrate hexahydrate were dissolved in $50 \mathrm{~mL}$ methanol respectively and by the same aging method at room temperature the pure ZIF-8 was collected.

\section{Synthesis of GO/Zn-Co-Ni LDHs composite}

The as-fabricated GO/ZIF-8 (80 mg weight) was added into $100 \mathrm{~mL}$ ethanol containing $200 \mathrm{mg}$ cobalt nitrate hexahydrate and $200 \mathrm{mg}$ nickel nitrate hexahydrate and then stirred for 3 minutes to mix well. The well mixed solution was transferred into round-bottom flask, and $120 \mathrm{mg}$ hexamethylenetetramine (HMT) was dissolved therein, and then it was maintained at $80{ }^{\circ} \mathrm{C}$ and refluxed for $1 \mathrm{~h}$. After cooling to room temperature, the GO/Zn-Co-Ni LDHs (GO-ZCN) was collected by centrifugation, washed several times with ethanol and water, and dried at $60{ }^{\circ} \mathrm{C}$. In order to compare well, when the mixed nitrate of $200 \mathrm{mg}$ cobalt nitrate hexahydrate and $200 \mathrm{mg}$ nickel nitrate hexahydrate were replaced by $400 \mathrm{mg}$ cobalt nitrate hexahydrate or $400 \mathrm{mg}$ nickel nitrate hexahydrate, GO/Zn-Co LDH (GO-ZC) and GO/Zn-Ni LDH (GO-ZN) were obtained with the same steps as the above operations respectively. 


\section{Materials characterization}

A Bruker D8 ADVANCE X-ray diffractometer was uesd to convey X-ray diffraction (XRD) data by using $\mathrm{Cu} \mathrm{K \alpha}$ radiation $(\lambda=1.5406 \AA)$ from 5 to $80(2 \theta)$ at a scanning rate of $7^{\circ} \mathrm{min}^{-1}$ with a voltage of $40 \mathrm{kV}$ and a current of $30 \mathrm{~mA}$. By the aid of a scanning electron microscope (SEM, JSM-7800) and high resolution transmission electron microscope (HRTEM, Jeol, JEM-2100HR), the morphology of all the samples showed very well.

\section{Electrochemical measurements}

At room temperature, all samples were putted into $1 \mathrm{M} \mathrm{KOH}$ aqueous electrolyte and performed its electrochemical properties by using a three-electrode system connected to electrochemical workstation CHI660C (Chen hua, Shanghai, China), and a Pt foil and a saturated calomel electrode (SCE) as the counter and reference electrodes respectively. ${ }^{1}$ According to a weight ratio of 80:10:10 in active material: carbon black: polytetrafluoroethylene (PTFE), the working electrode was prepared by mixing them together and coating onto the treated $\mathrm{Ni}$ foam (NF). After drying at $60{ }^{\circ} \mathrm{C}$ in vacuum drying oven for $12 \mathrm{~h}$, the prepared electrodes were pressed under a pressure of $10 \mathrm{MPa}$. By using cyclic voltammetry (CV), galvanostatic charge-discharge (GCD) and electrochemical impedance spectroscope (EIS), the electrochemical properties of the samples were measured. The formula used to calculate the specific capacitance of the electrode is as follows:

$$
C \mathrm{~s}=\frac{I \Delta \mathrm{t}}{m}
$$

In the equation, $C s\left(\mathrm{C} \mathrm{g}^{-1}\right), \Delta t(\mathrm{~s}), I(\mathrm{~A})$ and $m(\mathrm{~g})$ are the specific capacitance, the discharge time, the discharge current and the mass of the electrode materials, respectively.

\section{Package of the asymmetric supercapacitor}

In ASC, positive electrode, negative electrode, separator and semisolid electrolyte are corresponding to the prepared GO-ZCN, activated carbon (AC), polypropylene membrance and the PVA-KOH gel, respectively. An efficient charge storage and maximum potential window is builded on the charge balance theory of $\mathrm{Q}_{+}=\mathrm{Q}_{-}(\mathrm{Q}=$ 
$\mathrm{cm} \Delta \mathrm{V})$. According to this conclusion, the proper mass ratio of the positive and negative electrodes was around 4. On the other hand, the PVA-KOH gel electrolyte was made by a modified technique, which was reported in our previous work. ${ }^{2} 60 \mathrm{~mL}$ of deionized water solution containing polyvinyl alcohol was stirred at $85{ }^{\circ} \mathrm{C}$ about $1.5 \mathrm{~h}$ with a transparent state.After cooling down, the prepared $\mathrm{KOH}$ solution dripped under stirring and then the mixture was kept stirring $0.5 \mathrm{~h}$ until a uniform solution was obtained. In the ASC, the negative electrode made up from AC was employed the same operations with the positive electrode to achieve. The following step was putting all the electrode materials into the prepared PVA-KOH gel electrolyte for about 3 to 5 minutes and then taking them out for assembling. The soaked positive and negative electrodes were separated by a separator (polypropylene membrance), and they were all enclosed in a current collector. After these operations were completed, a solid supercapacitor was assembled. Finally, the experimental data were tested by the way of a two-electrode system at the electrochemical workstation. The formulas below are used to calculate the energy density $(\mathrm{E})$ and power density $(\mathrm{P})$ of the equipment:

$$
\begin{aligned}
& C=\frac{I \Delta \mathrm{t}}{m \Delta V} \\
& E=\frac{0.5 C(\Delta V)^{2}}{3.6} \\
& P=\frac{3600 E}{\Delta t}
\end{aligned}
$$

The meanings of $C\left(\mathrm{~F} \mathrm{~g} \mathrm{~g}^{-1}\right), \Delta t(\mathrm{~s}), \Delta V(\mathrm{~V})$ and $I(\mathrm{~A})$ are the specific capacitance, the discharge time, the potential window and the discharge current of an asymmetric capacitor respectively, and $m(\mathrm{~g})$ is the mass of active materials. 


\section{Supplementary results}

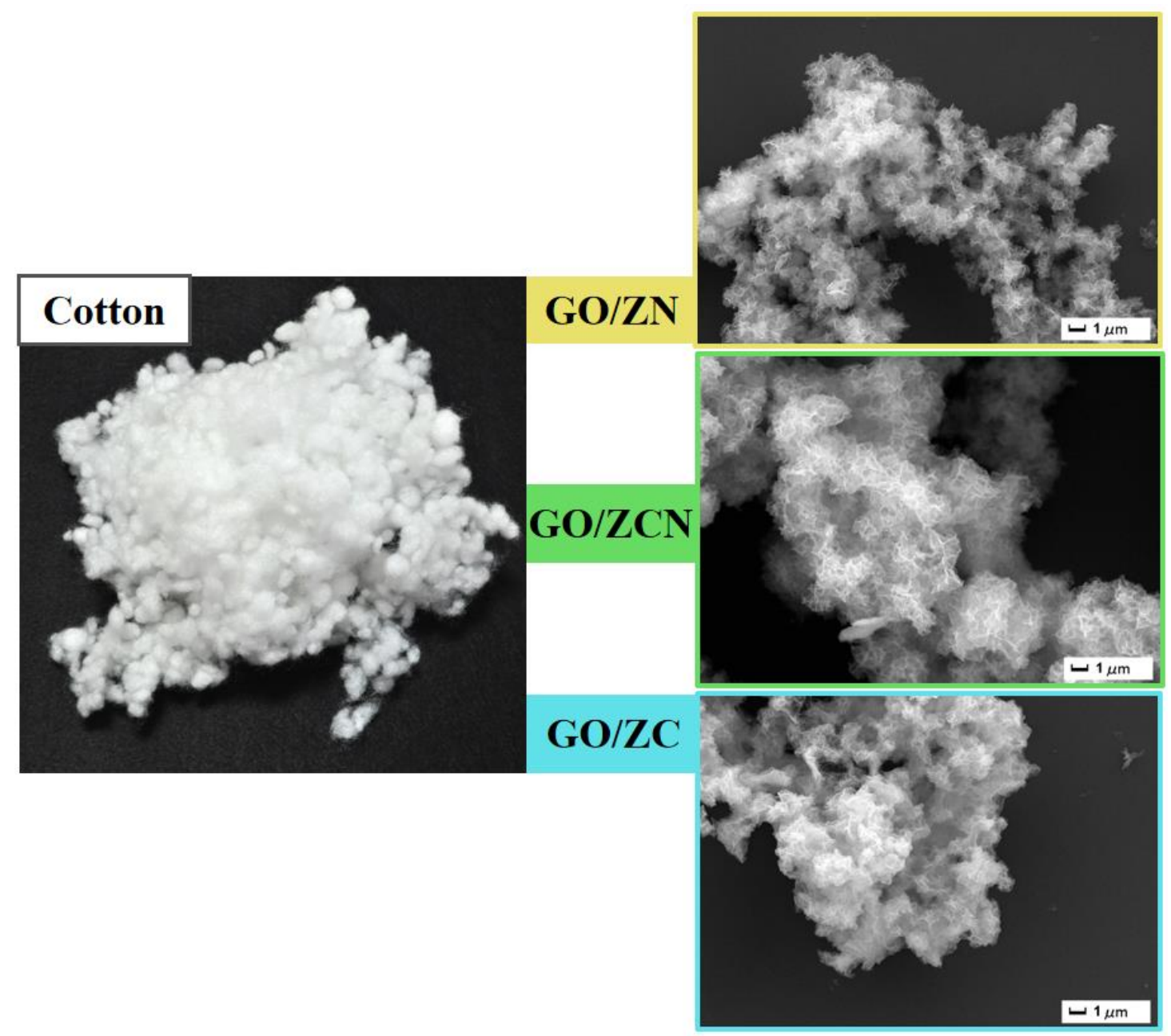

Figure S1 The comparison pictures of the fluffy cotton-like structure of the three samples. 

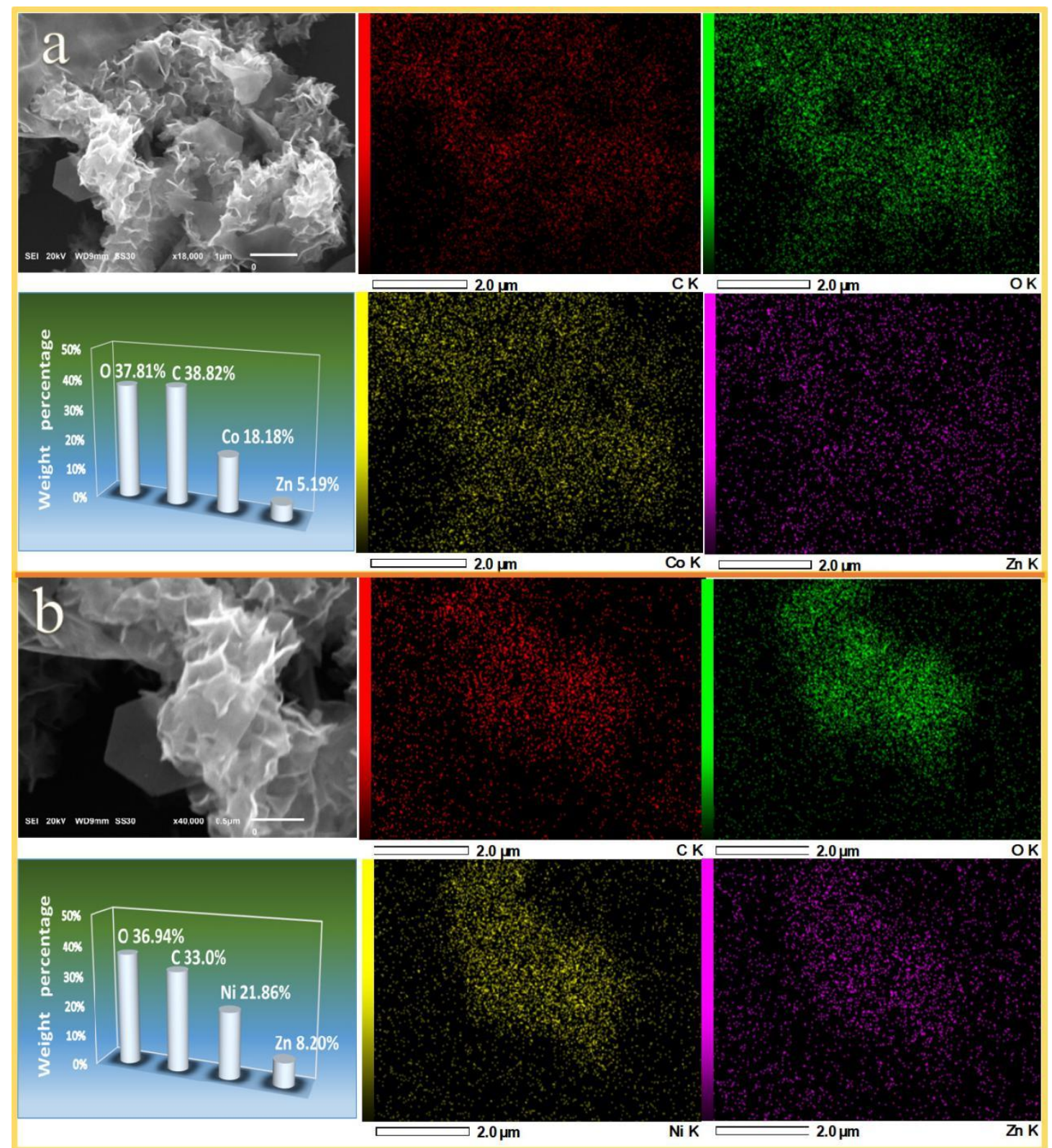

Figure S2 (a) Elemental mapping and quantitative resultes of the GO/ZC ,(b) elemental mapping and quantitative resultes of the GO/ZN. 

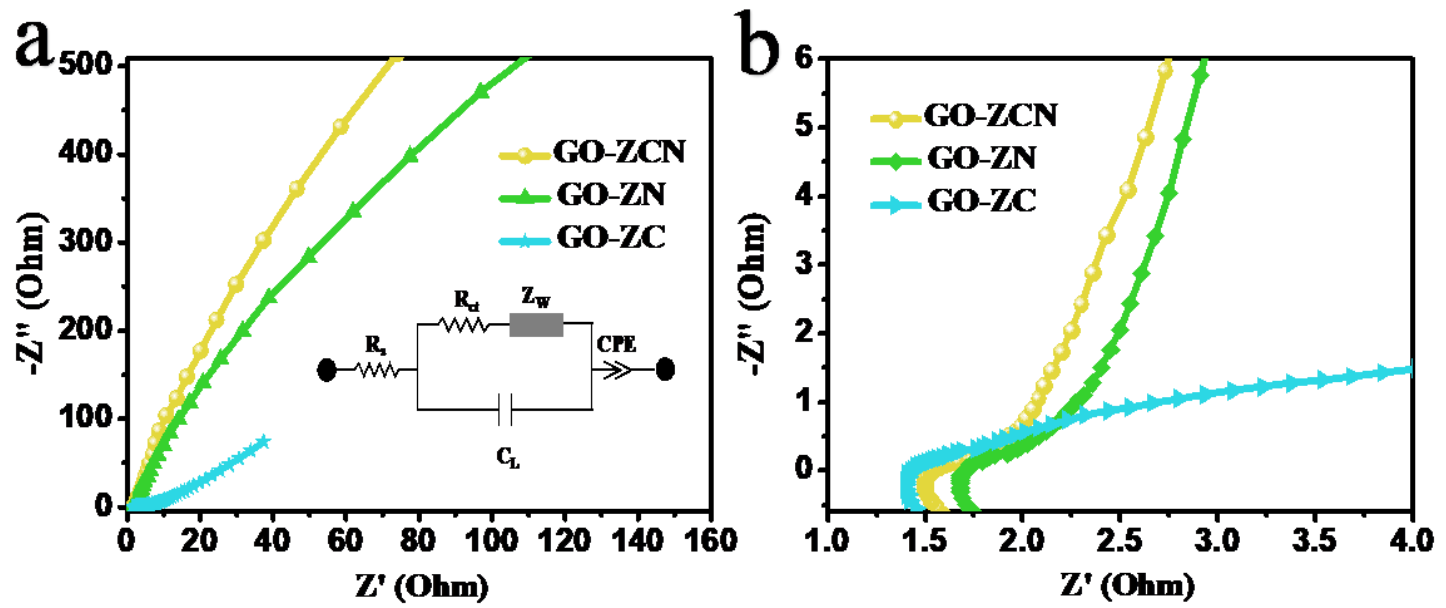

Figure S3 (a) Nyquist impedance plots of GO/ZCN, GO/ZN and GO/ZC. (b) The impedance at a high-frequency region of GO/ZCN, GO/ZN and GO/ZC. 

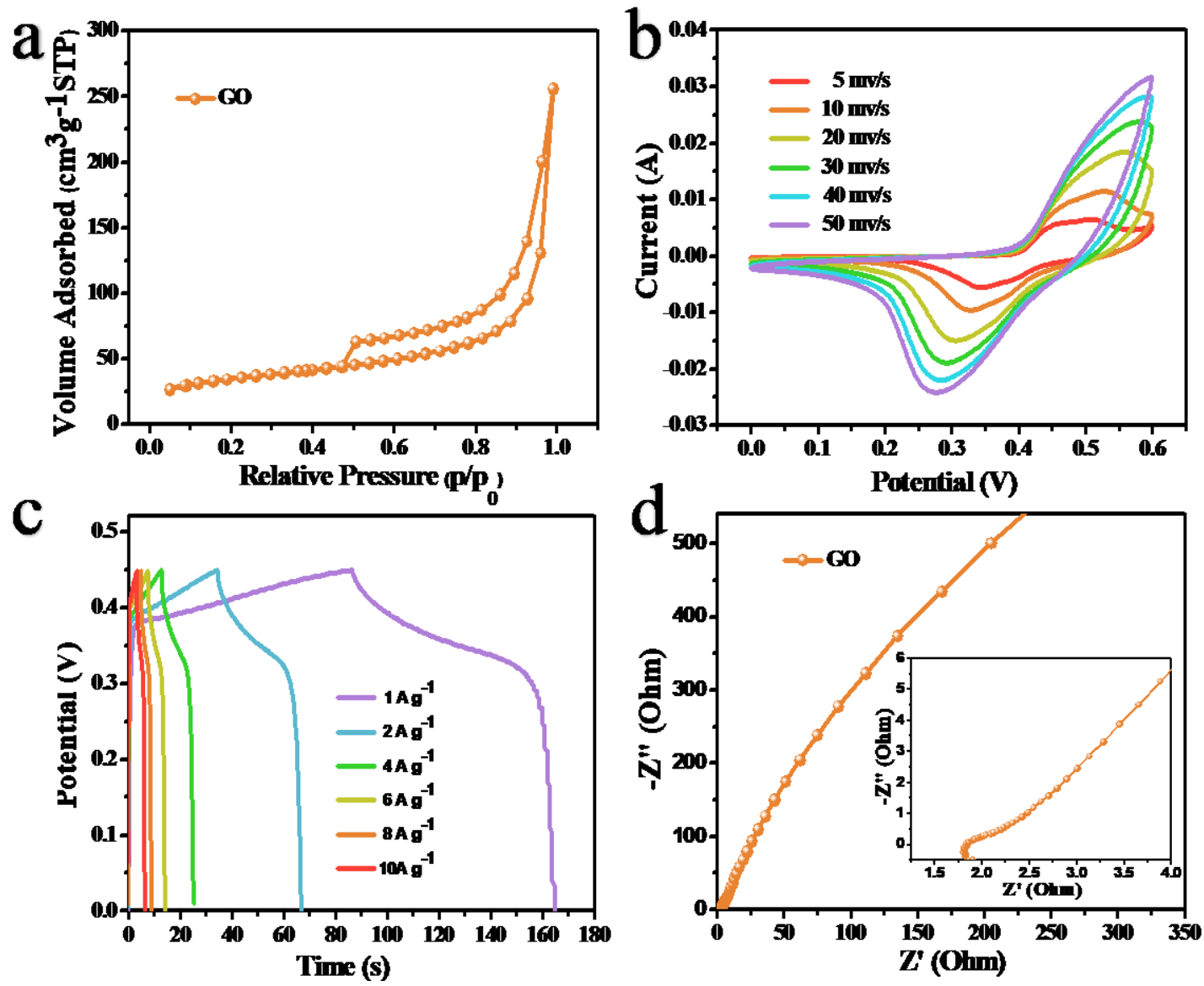

Figure S4 (a) $\mathrm{N}_{2}$ adsorption-desorption isotherm curve of GO, (b) CV curves of GO at different scan rates, (c) GCD curves of GO at different current densities, (d) Nyquist impedance plot of GO, illustrations: partial enlarged detail at high frequency zone. 


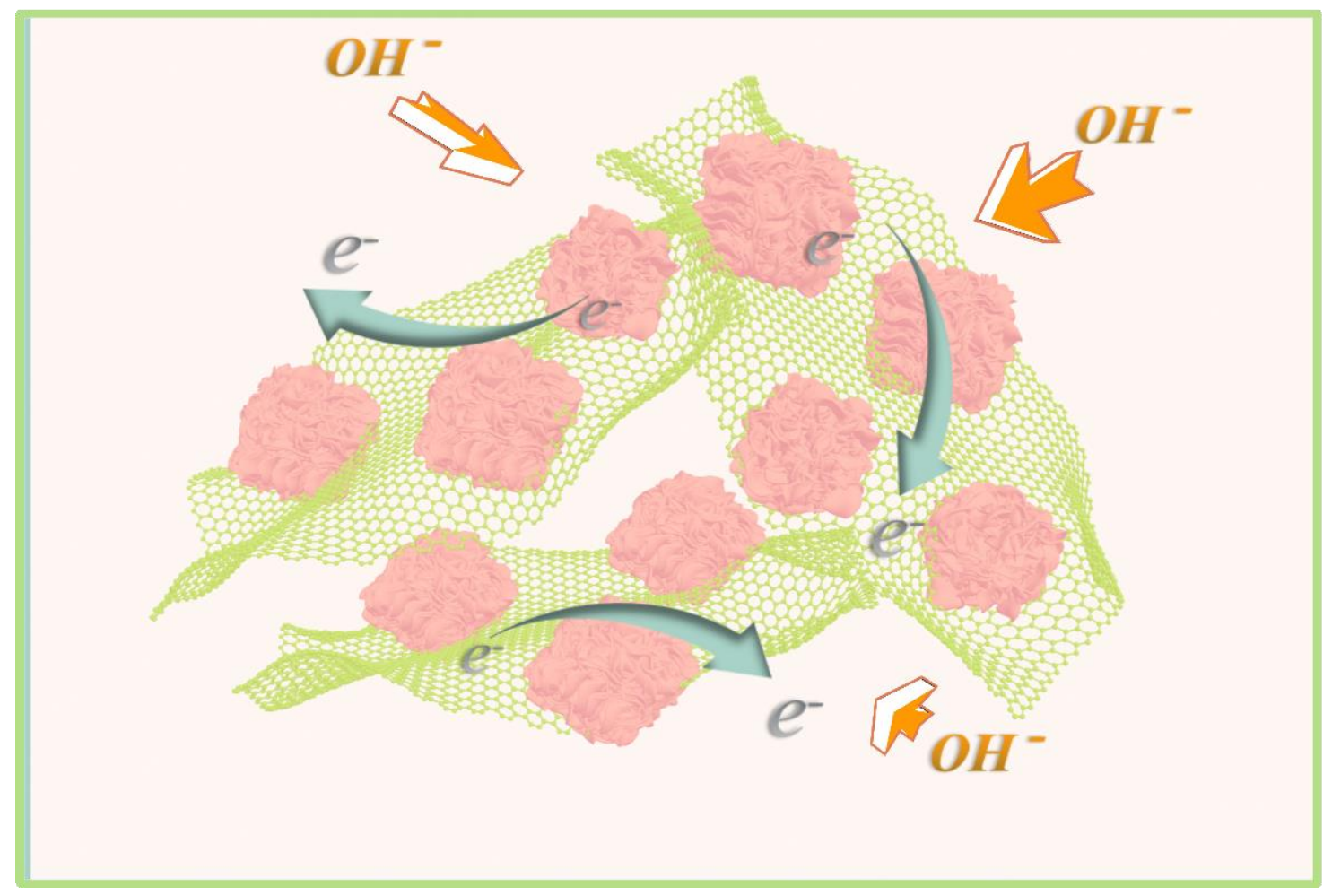

Figure S5 Electron transport and ion diffusion mechanism diagram of positive active material in GO/ZCN//AC ASC. 

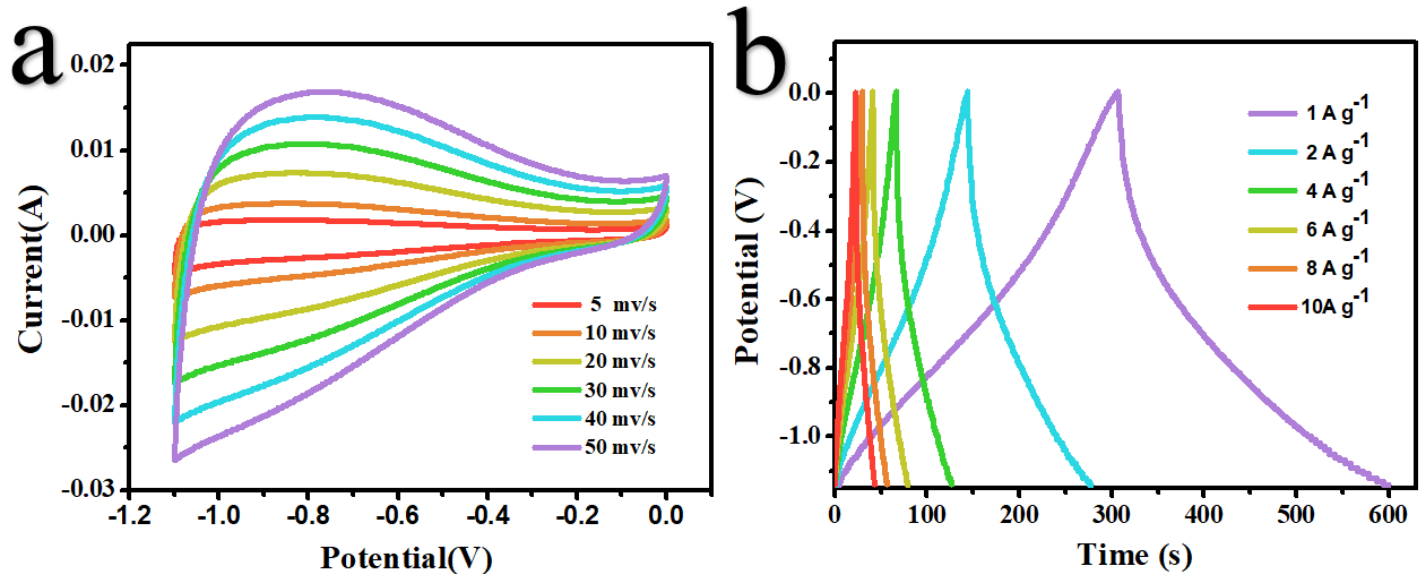

Figure S6 (a) CV curves of AC at different scan rates, (b) GCD curves of AC at different current densities. 

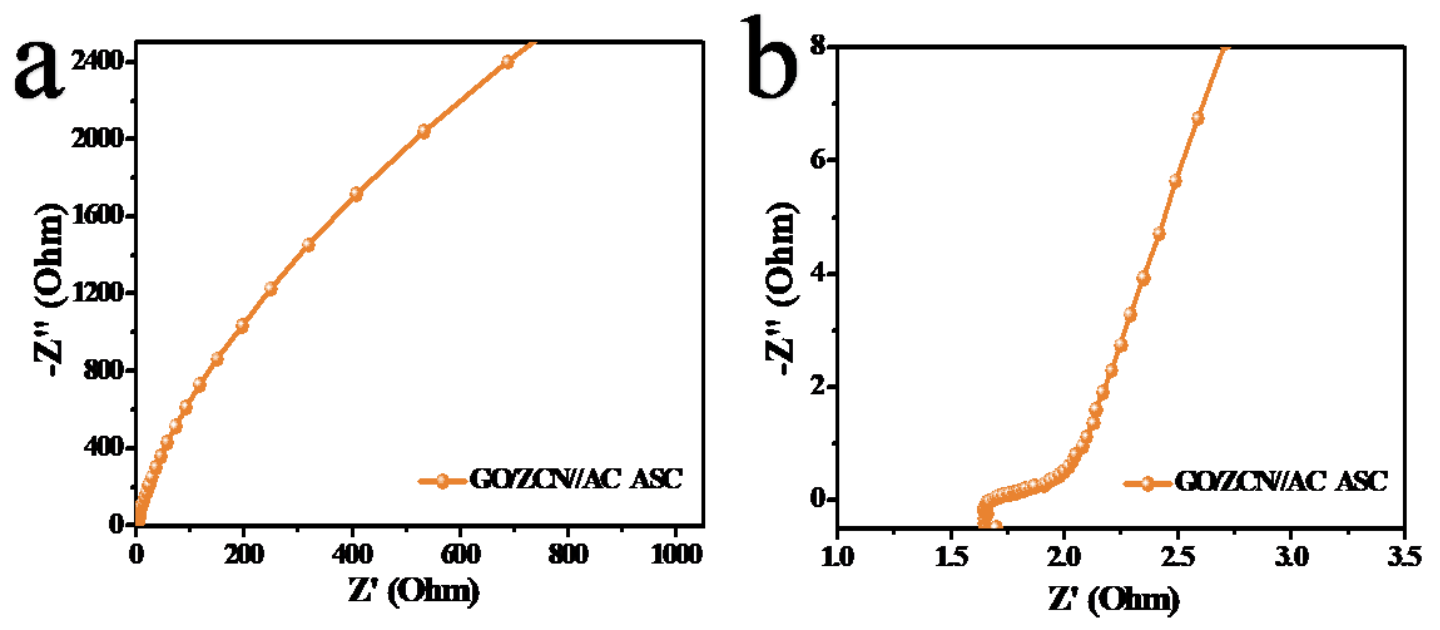

Figure S7 (a) Nyquist impedance plot of the GO/ZCN//AC ASC, (b) The impedance at a high-frequency region of the GO/ZCN//AC ASC. 


\section{REFERENCES}

(S1) Zhou, S.; Hao, C.; Wang, J.; Wang, X.; Gao, H. Metal-organic framework templated synthesis of porous $\mathrm{NiCo}_{2} \mathrm{O}_{4} / \mathrm{ZnCo}_{2} \mathrm{O}_{4} / \mathrm{Co}_{3} \mathrm{O}_{4}$ hollow polyhedral nanocages and their enhanced pseudocapacitive properties. Chem. Eng. J. 2018, 351, 74-84. DOI 10.1016/j.cej.2018.06.070.

(S2) Zhou, S.; Ye, Z.; Hu, S.; Hao, C.; Wang, X.; Huang, C.; Wu, F. Designed formation of $\mathrm{Co}_{3} \mathrm{O}_{4} / \mathrm{ZnCo}_{2} \mathrm{O}_{4} / \mathrm{CuO}$ hollow polyhedral nanocages derived from zeolitic imidazolate framework-67 for high-performance supercapacitors. Nanoscale 2018, 10, 15771-15781. DOI 10.1039/C8NR05138K. 